\title{
Generalized half-logistic Poisson distributions
}

\author{
Mustapha Muhammad ${ }^{1, a}$ \\ ${ }^{a}$ Department of Mathematical Science, Bayero University Kano, Nigeria
}

\begin{abstract}
In this article, we proposed a new three-parameter distribution called generalized half-logistic Poisson distribution with a failure rate function that can be increasing, decreasing or upside-down bathtub-shaped depending on its parameters. The new model extends the half-logistic Poisson distribution and has exponentiated half-logistic as its limiting distribution. A comprehensive mathematical and statistical treatment of the new distribution is provided. We provide an explicit expression for the $r^{\text {th }}$ moment, moment generating function, Shannon entropy and Rényi entropy. The model parameter estimation was conducted via a maximum likelihood method; in addition, the existence and uniqueness of maximum likelihood estimations are analyzed under potential conditions. Finally, an application of the new distribution to a real dataset shows the flexibility and potentiality of the proposed distribution.
\end{abstract}

Keywords: half-logistic Poisson, moments, entropy, maximum likelihood estimates

\section{Introduction}

Lifetime data may exhibit a decreasing, increasing, or a bathtub failure rate function when modeling and analyzing random phenomena. This arises in several areas of studies, such as biomedical studies, reliability, actuarial science, computer science, demography, and engineering. There are several lifetime models that have been used successfully for to analyze lifetime data in practical applications such as exponential, Weibull, Gompertz, generalized exponential, and half-logistic. For example, exponential distribution can accommodate lifetime data with a decreasing density despite having only a constant failure rate function. Gompertz distribution has a decreasing and unimodal density but has an increasing failure rate function. However, these distributions are unable to accommodate lifetime data with a non-monotone failure rate such as the bathtub or upside-down bathtub. Many researchers have attempted to provide several methods to generate new lifetime models with the ability to fit data with monotone or non-monotone failure rate to overcome these problems. These methods include the generalization of a distribution by exponentiation procedure.

Method of exponentiation is an of the important and commonly used techniques to add a parameter to a lifetime model, the new model becomes more flexible and can accommodate both monotones as well as non-monotone failure rate functions. For example, Kuş (2007) proposed en exponential Poisson (EP) distribution that possesses a decreasing failure rate function; however, Barreto-Souza and Cribari-Neto (2009) proposed generalized exponential Poisson (GEP) by exponentiation of the EP. The GEP distribution can accommodate decreasing, increasing and upside down bathtub failure rates. Similarly, Adamidis and Loukas (1998) introduced exponential geometric distribution, while Silva et al. (2010) come up with the generalized exponential geometric distribution. Tahmasbi and

\footnotetext{
${ }^{1}$ Department of Mathematical Science, Bayero University Kano, Nigeria.

E-mail: mmmahmoud12@sci.just.edu.jo,mmuhammad.mth@buk.edu.ng

Published 31 July 2017 / journal homepage: http://csam.or.kr

(c) 2017 The Korean Statistical Society, and Korean International Statistical Society. All rights reserved.
} 
Rezaei (2008) introduced exponential-logarithmic distribution similarly, Pappas et al. (2015) proposed a generalized exponential-logarithmic. Silva and Cordeiro (2015) introduced a new class of distribution called the BurrXII Poisson distribution and recently Muhammad (2016a) proposed generalized BurrXII Poisson distribution. To read more about exponentiated distributions see Ali et al. (2007) and Raja and Mir (2011). A new two-parameter distribution known as the half-logistic Poisson (HLP) distribution was also introduced by Muhammad and Yahaya (2017) using the procedure followed by Kuş (2007) and Chung and Kang (2014).

The cumulative distribution function (cdf) of the HLP distribution is given by

$$
G(x)=\left(\frac{1-e^{-\lambda\left(\frac{1-e^{-\alpha x}}{1+e^{-\alpha x}}\right)}}{1-e^{-\lambda}}\right),
$$

with $x>0, \alpha>0$, and $\lambda>0$. The HLP distribution can accommodate data with increasing or decreasing hazard functions.

The first motivation of this study is to propose a new three-parameter probability distribution with increasing, decreasing and upside down bathtub-shaped failure functions. The second motivation of this study is to find the association of the density functions and hazard rate functions in practical applications. Last, we are motivated because the exponentiation method provides additional flexibility to a model especially in both the density and failure rate functions. The HLP distribution cannot fit data set with non-monotone failure rate functions; therefore, we hope that the new distribution that is the generalized half-logistic Poisson (GHLP) will provide solutions to many problems in various fields in practical applications.

The rest of the paper is arranged as follows, in Section 2 we provide the density of the GHLP and consider some important mathematical and statistical properties. Section 3 discusses the maximum likelihood estimate (MLE) and a simulation study. Section 4 provides applications of the new model to a real data set Section 5 provides the conclusions.

\section{The proposed model}

The cumulative distribution function of the GHLP distribution with parameters $\alpha, \beta, \lambda>0$ is given by

$$
F(x)=\left(1-e^{-\lambda\left(\frac{1-e^{-\alpha x}}{1+e^{-\alpha x}}\right)}\right)^{\beta}\left(1-e^{-\lambda}\right)^{-\beta}
$$

where the corresponding probability density and hazard rate functions are given by

$$
\begin{aligned}
& f(x)=\frac{2 \alpha \beta \lambda e^{-\alpha x}}{\left(1-e^{-\lambda}\right)^{\beta}\left(1+e^{-\alpha x}\right)^{2}}\left(1-e^{-\lambda\left(\frac{1-e^{-\alpha x}}{1+e^{-\alpha x}}\right)}\right)^{\beta-1} e^{-\lambda\left(\frac{1-e^{-\alpha x}}{1+e^{-\alpha x}}\right)}, \\
& h(x)=\frac{2 \alpha \beta \lambda e^{-\alpha x}\left(1-e^{-\lambda\left(\frac{1-e^{-\alpha x}}{1+e^{-\alpha x}}\right)}\right)^{\beta-1} e^{-\lambda\left(\frac{1-e^{-\alpha x}}{1+e^{-\alpha x}}\right)}}{\left(1+e^{-\alpha x}\right)^{2}\left(\left(1-e^{-\lambda}\right)^{\beta}-\left(1-e^{-\lambda\left(\frac{1-e^{-\alpha x}}{1+e^{-\alpha x}}\right)}\right)^{\beta}\right)},
\end{aligned}
$$

respectively. The limiting distribution given by (2.1) when $\lambda \rightarrow 0^{+}$is $\lim _{\lambda \rightarrow 0^{+}} F(x)=\left(\left(1-e^{-\alpha x}\right) /\right.$ $\left.\left(1+e^{-\alpha x}\right)\right)^{\beta}$ for $\beta>0$, which is the cdf of the exponentiated half-logistic distribution.

Theorem 1. The probability density function ( $p d f)$ given by $(2.2)$ is decreasing function for $\beta \leq 1$. 

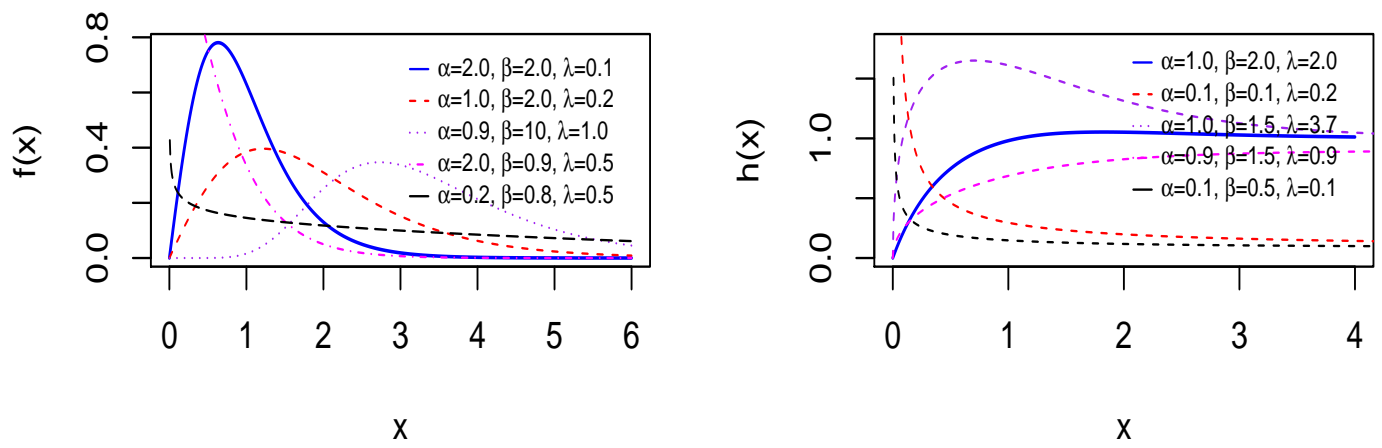

Figure 1: Plots of the probability density function and hazard function of the generalized half-logistic Poisson for different values of parameters.

Proof: We obtained the first derivative of $\log f(x)$ as

$$
\frac{\partial \log f(x)}{\partial x}=-\alpha-\frac{2 \alpha e^{-\alpha x}}{1-e^{-\alpha x}}+\frac{2 \alpha \lambda(\beta-1) e^{-\alpha x} e^{-\lambda\left(\frac{1-e^{-\alpha x}}{1+e^{-\alpha x}}\right)}}{\left(1+e^{-\alpha x}\right)^{2}\left(1-e^{-\lambda\left(\frac{1-e^{-\alpha x}}{1+e^{-\alpha x}}\right)}\right)}-\frac{2 \alpha \lambda e^{-\alpha x}}{\left(1+e^{-\alpha x}\right)^{2}}
$$

thus, for $\beta \leq 1, \log f^{\prime}(x)<0$.

Figure 1 below provide the plot of the pdf $(f(x))$ of the GHLP and illustrated that the pdf can be unimodal function for $\beta>1$.

The limiting behavior of the pdf given by (2.2) are: for $\beta<1, \lim _{x \rightarrow 0} f(x)=\infty$; for $\beta=1$, $\lim _{x \rightarrow 0} f(x)=\alpha \lambda /\left\{2\left(1-e^{-\lambda}\right)\right\}$; for $\beta>1, \lim _{x \rightarrow 0} f(x)=0$ and $\lim _{x \rightarrow \infty} f(x)=0$ for all $\beta>0$. The limiting behavior of the hazard rate function given by (2.3) are: for $\beta<1, \lim _{x \rightarrow 0} h(x)=\infty$; for $\beta=1, \lim _{x \rightarrow 0} h(x)=\alpha \lambda /\left\{2\left(1-e^{-\lambda}\right)\right\}$ and for $\beta>1, \lim _{x \rightarrow 0} h(x)=0$. Figure 1 below shows that the hazard rate function $(h(x))$ of the GHLP given by (2.3) can be decreasing, increasing or unimodal functions.

The $p^{\text {th }}$-quantile function of the GHLP can easily be derived by inverting (2.1) as

$$
\xi(p)=-\alpha^{-1} \log \left(\frac{\lambda+\log \left(1-p^{\frac{1}{\beta}}\left(1-e^{-\lambda}\right)\right)}{\lambda-\log \left(1-p^{\frac{1}{\beta}}\left(1-e^{-\lambda}\right)\right)},\right.
$$

therefore, we can find the numerical values of the median and other percentiles of $X$ with (2.5), also the median of $X$ can be obtained as $\xi(0.5)$. Moreover, equation (2.5) can be used to generate random data distributed $\operatorname{GHLP}(\alpha, \beta, \lambda)$ by setting $p \sim U(0,1)$, where $U(0,1)$ is the uniform distribution.

\subsection{Moments}

Here, we provide the following lemma which is very useful in computations of several important properties of the GHLP. first we recall that, $B_{k d}(a, b)=\left(\partial^{k+d} B(a, b)\right) /\left(\partial a^{k} \partial b^{d}\right)$ for $k+a>0, b+d>0$, where $B(a, b)=\int_{0}^{1} u^{a-1}(1-u)^{b-1} d u$ is a beta function. 
Table 1: Numerical values of $J\left(J_{1}, J_{2}, J_{3}, J_{4}, J_{5}, J_{6}\right)$ for some values of $J_{1}, J_{2}, J_{3}, J_{4}, J_{5}$, and $J_{6}$

\begin{tabular}{ccccccc|ccccccc}
\hline \hline$J_{1}$ & $J 2$ & $J 3$ & $J 4$ & $J 5$ & $J 6$ & $J\left(J_{1}, J_{2}, J_{3}, J 4, J 5, J 6\right)$ & $J 1$ & $J 2$ & $J 3$ & $J 4$ & $J 5$ & $J 6$ & $J\left(J_{1}, J_{2}, J_{3}, J_{4}, J_{5}, J_{6}\right)$ \\
\hline 0.5 & 1.2 & 2.1 & 2.3 & 0.6 & 3.0 & 0.01595 & 0.5 & 0.2 & 0.1 & 0.3 & 0.6 & 0.3 & 7.24441 \\
0.6 & 2.2 & 1.1 & 4.3 & 3.6 & 1.3 & 0.00392 & 0.1 & 2.2 & 4.1 & 3.3 & 3.6 & 1.5 & 0.00065 \\
3.0 & 2.0 & 4.1 & 3.3 & 6.0 & 5.0 & 0.00150 & 1.0 & 6.0 & 1.0 & 3.0 & 2.0 & 1.0 & 0.00024 \\
1.0 & 1.0 & 1.0 & 1.0 & 1.0 & 1.0 & 0.23417 & 1.5 & 1.0 & 1.0 & 2.5 & 1.0 & 0.5 & 0.34307 \\
1.5 & 7.0 & 1.0 & 2.5 & 1.0 & 1.5 & 0.00018 & 10 & 2.0 & 2.0 & 5.0 & 1.0 & 5.0 & 14.2023 \\
10 & 2.0 & 12 & 5.0 & 11 & 15 & 0.00123 & 8.0 & 5.0 & 2.0 & 1.0 & 9.0 & 1.0 & 0.00240 \\
\hline \hline
\end{tabular}
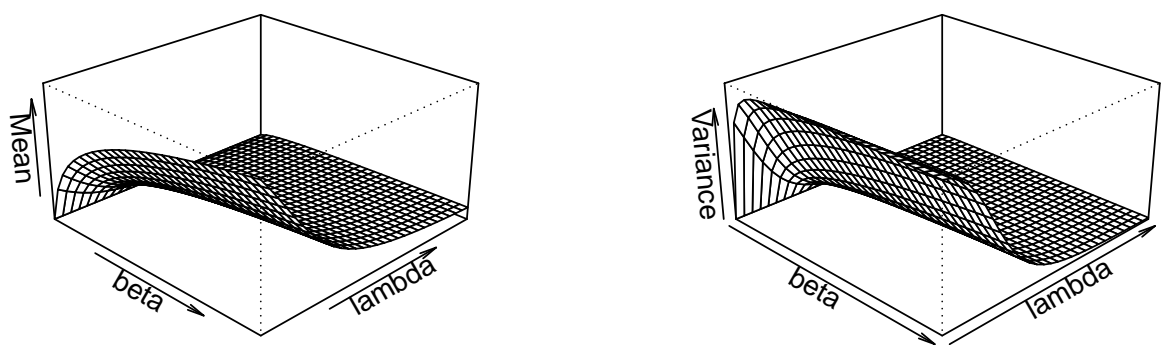

Figure 2: Plots of the mean and variance of generalized half-logistic Poisson for $\alpha=1.5$.

Lemma 1. For $J_{n}, n=1,2, \ldots, 6$, and $J_{n} \in \mathbb{R}$, let,

$$
J\left(J_{1}, J_{2}, J_{3}, J_{4}, J_{5}, J_{6}\right)=\int_{0}^{\infty} \frac{x^{J_{1}} e^{-J_{2} x}\left(1-e^{-\alpha x}\right)^{J_{3}}}{\left(1+e^{-\alpha x}\right)^{J_{4}}}\left(1-e^{-\lambda\left(\frac{1-e^{-\alpha x}}{1+e^{-\alpha x}}\right)}\right)^{J_{5}} e^{-J_{6}\left(\frac{1-e^{-\alpha x}}{1+e^{-\alpha x}}\right)} d x
$$

then,

$$
J\left(J_{1}, J_{2}, J_{3}, J_{4}, J_{5}, J_{6}\right)=\sum_{i=0}^{\infty} \sum_{k, l=0}^{\infty} \xi_{i, k, l} B_{0 J_{1}}\left(J_{3}+k+1, \frac{J_{2}}{\alpha}+l\right)
$$

where $\xi_{i, k, l}=\left(\begin{array}{c}J_{5} \\ i\end{array}\right)\left(\begin{array}{c}\left(J_{4}+k\right) \\ l\end{array}\right)\left\{(-1)^{i+k+J_{1}}\left(\lambda i+J_{6}\right)^{k}\right\} /\left\{\alpha^{J_{1}+1} k !\right\}$.

Proof: See Appendix A.

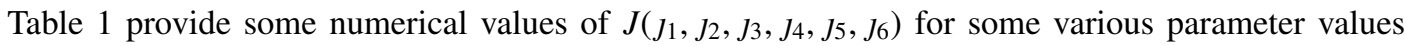
computed using R software.

Various characteristics and features of a distribution can be analyzed through its moments such as mean, variance, moment generating function, etc. If $X$ follows the GHLP distribution, then, the $r^{\text {th }}$ moment of $X$ can be obtained by considering Lemma 1 as follows:

$$
E\left(X^{r}\right)=\frac{2 \alpha \beta \lambda}{\left(1-e^{-\lambda}\right)^{\beta}} \int_{0}^{\infty} \frac{x^{r} e^{-\alpha x}}{\left(1+e^{-\alpha x}\right)^{2}}\left(1-e^{-\lambda\left(\frac{1-e^{-\alpha x}}{1+e^{-\alpha x}}\right)}\right)^{\beta-1} e^{-\lambda\left(\frac{1-e^{-\alpha x}}{1+e^{-\alpha x}}\right)} d x,
$$

thus,

$$
E\left(X^{r}\right)=\frac{2 \alpha \beta \lambda}{\left(1-e^{-\lambda}\right)^{\beta}} J(r, \alpha, 0,2, \beta-1, \lambda) .
$$

Therefore, the moments of GHLP can easily be computed using mathematical software such as MAT$L A B, M A T H E M A T I C A$, and $R$. Figure 2 provide the plots of the mean $\mu$ and variance $\sigma^{2}$ of the GHLP 

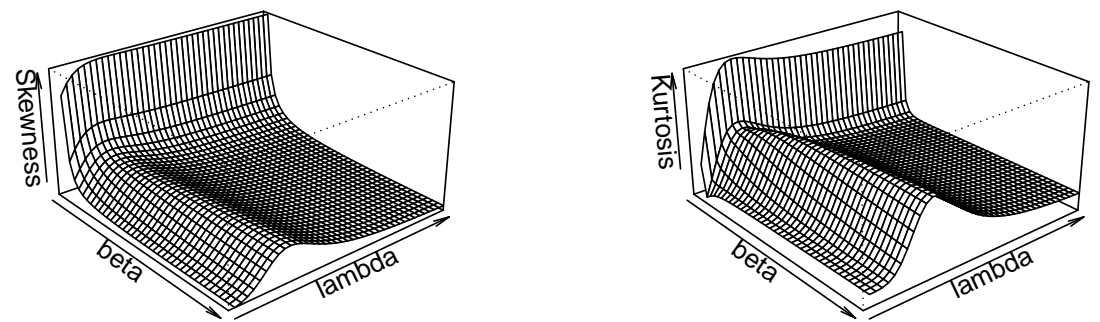

Figure 3: Plots of the skewness and kurtosis of generalized half-logistic Poisson for $\alpha=0.5$.

for $\alpha=1.5$, it is clear that both the mean and variance are increasing as $\beta$ increases and decreasing as $\lambda$ increases.

The moment generating function of the GHLP distribution can be computed directly using $M_{X}(t)=$ $E\left(e^{t X}\right)$ which can be expanded to

$$
M_{X}(t)=\sum_{r=0}^{\infty} \frac{t^{r}}{r !} E\left(X^{r}\right)
$$

thus, by putting (2.9) in (2.11) we have

$$
M_{X}(t)=\sum_{r=0}^{\infty} \frac{2 \alpha \beta \lambda t^{r}}{\left(1-e^{-\lambda}\right)^{\beta} r !} J(r, \alpha, 0,2, \beta-1, \lambda) .
$$

One of the alternative measures for the skewness and kurtosis of a distribution are the Bowley skewness (B) and Moor's kurtosis (M) defined by $B=[\xi(3 / 4)+\xi(1 / 4)-2 \xi(2 / 4)] /[\xi(3 / 4)-\xi(1 / 4)]$ and $M=[\xi(3 / 8)-\xi(1 / 8)+\xi(7 / 8)-\xi(5 / 8)] /[\xi(6 / 8)-\xi(2 / 8)]$, respectively, where $\xi(\cdot)$ is given by (2.5). Figure 3 is the plots of the Bowley skewness and Moor's kurtosis of the GHLP for $\alpha>0$. It is clear that both the skewness and kurtosis are decreasing in $\beta$ and unimodal function in $\lambda$.

\subsection{Entropy}

Entropy is defined as a measure of uncertainty of a random variable. Here, we consider the two most important entropies known as the Shannon and Rényi entropies. The Shannon entropy is defined by $E[-\log f(x)]$. For a random variable $X$ with GHLP, the Shannon entropy can be computed by considering the Proposition 1 as follows.

Proposition 1. Let $X$ be a random variable with pdf given by (2.2), then,

$$
\begin{aligned}
E\left(\log \left(1+e^{-\alpha X}\right)\right) & =\left.\frac{2 \alpha \beta \lambda}{\left(1-e^{-\lambda}\right)^{\beta}} \frac{\partial}{\partial t} J(0, \alpha, 0,2-t, \beta-1, \lambda)\right|_{t=0}, \\
E\left(\log \left(1-e^{-\lambda\left(\frac{1-e^{-\alpha X}}{1+e^{-\alpha X}}\right)}\right)\right) & =\left.\frac{2 \alpha \beta \lambda}{\left(1-e^{-\lambda}\right)^{\beta}} \frac{\partial}{\partial t} J(0, \alpha, 0,2, \beta+t-1, \lambda)\right|_{t=0}, \\
E\left(\frac{1-e^{-\alpha X}}{1+e^{-\alpha X}}\right) & =\frac{2 \alpha \beta \lambda}{\left(1-e^{-\lambda}\right)^{\beta}} J(0, \alpha, 1,3, \beta-1, \lambda) .
\end{aligned}
$$

where $J(\cdot, \cdot, \cdot, \cdot, \cdot, \cdot)$ is given by (2.7). 
Hence,

$$
\begin{aligned}
E[-\log f(X)]= & -\log \left(\frac{2 \alpha \beta \lambda}{\left(1-e^{-\lambda}\right)^{\beta}}\right)+\alpha E(X)+\left.\frac{4 \alpha \beta \lambda}{\left(1-e^{-\lambda}\right)^{\beta}} \frac{\partial}{\partial t} J(0, \alpha, 0,2-t, \beta-1, \lambda)\right|_{t=0} \\
& -\left.\frac{2 \alpha \beta \lambda(\beta-1)}{\left(1-e^{-\lambda}\right)^{\beta}} \frac{\partial}{\partial t} J(0, \alpha, 0,2, \beta+t-1, \lambda)\right|_{t=0}+\frac{2 \alpha \beta \lambda^{2}}{\left(1-e^{-\lambda}\right)^{\beta}} J(0, \alpha, 1,3, \beta-1, \lambda) .
\end{aligned}
$$

The Rényi entropy of a random variable $X$ is defined by $I_{R(\rho)}=1 /(1-\rho) \log \left[\int_{0}^{\infty} f(x)^{\rho} d x\right]$, where $\rho>0$ and $\rho \neq 1$. The Rényi entropy of $X$ that has GHLP is obtain as follows.

$$
\int_{0}^{\infty} f^{\rho}(x) d x=\int_{0}^{\infty} \frac{2^{\rho} \alpha^{\rho} \beta^{\rho} \lambda^{\rho} e^{-\alpha \rho x} e^{-\lambda \rho\left(\frac{1-e^{-\alpha x}}{1+e^{-\alpha x}}\right)}}{\left(1-e^{-\lambda}\right)^{\beta \rho}\left(1+e^{-\alpha x}\right)^{2 \rho}}\left(1-e^{-\lambda\left(\frac{1-e^{-\alpha x}}{1+e^{-\alpha x}}\right)}\right)^{\rho(\beta-1)} d x,
$$

by applying Lemma 1 the integral become

$$
\int_{0}^{\infty} f^{\rho}(x) d x=\frac{2^{\rho} \alpha^{\rho} \beta^{\rho} \lambda^{\rho}}{\left(1-e^{-\lambda}\right)^{\beta \rho}} J(0, \alpha \rho, 0,2 \rho, \rho(\beta-1), \lambda \rho) .
$$

Hence, the Rényi entropy of $X$ is

$$
I_{R(\rho)}=\frac{1}{1-\rho} \log \left[\frac{2^{\rho} \alpha^{\rho} \beta^{\rho} \lambda^{\rho}}{\left(1-e^{-\lambda}\right)^{\beta \rho}} J(0, \alpha \rho, 0,2 \rho, \rho(\beta-1), \lambda \rho)\right] .
$$

\section{Estimation}

In this section, we discuss MLEs, one of the most popular and common methods used in inference. In this method the approximate MLEs are obtained either analytically or numerically using some mathematical packages. Let $X_{i}(i=1,2, \ldots, n)$ be a random sample of size $\mathrm{n}$ from the GHLP distribution with observed values $x_{1}, x_{2}, x_{3}, \ldots, x_{n}$. The $\log$ likelihood function, i.e. $\log \ell(\theta)$ for complete data set of the GHLP distribution is given by

$$
\begin{aligned}
\log \ell(\theta)= & n \log 2+n \log \alpha+n \log \beta+n \log \lambda-n \beta \log \left(1-e^{-\lambda}\right)-\alpha \sum_{i=1}^{n} x_{i} \\
& -2 \sum_{i=1}^{n} \log \left(1+e^{-\alpha x_{i}}\right)+(\beta-1) \sum_{i=1}^{n} \log \left(1-e^{-\lambda\left(\frac{1-e^{-\alpha x_{i}}}{1+e^{-\alpha x_{i}}}\right)}\right)-\lambda \sum_{i=1}^{n}\left(\frac{1-e^{-\alpha x_{i}}}{1+e^{-\alpha x_{i}}}\right) .
\end{aligned}
$$

Hence, the MLEs of $\theta=(\alpha, \beta, \lambda)^{T}$, say $\hat{\theta}=(\hat{\alpha}, \hat{\beta}, \hat{\lambda})^{T}$ is the solution of the nonlinear equations (3.2)-(3.4).

$$
\begin{aligned}
& \frac{\partial \ell}{\partial \alpha}=\frac{n}{\alpha}-\sum_{i=1}^{n} x_{i}-\sum_{i=1}^{n} \frac{2 x_{i} e^{-\alpha x_{i}}}{1+e^{-\alpha x_{i}}}+\sum_{i=1}^{n} \frac{2(\beta-1) \lambda x_{i} e^{-\alpha x_{i}} e^{-\lambda\left(\frac{1-e^{-\alpha x_{i}}}{1+e^{-\alpha x_{i}}}\right)}}{\left(1+e^{-\alpha x_{i}}\right)^{2}\left(1-e^{-\lambda\left(\frac{1-e^{-\alpha x_{i}}}{1+e^{-\alpha x_{i}}}\right)}\right)}-\sum_{i=1}^{n} \frac{2 \lambda x_{i} e^{-\alpha x_{i}}}{\left(1+e^{-\alpha x_{i}}\right)^{2}}=0, \\
& \frac{\partial \ell}{\partial \beta}=\frac{n}{\beta}-n \log \left(1-e^{-\lambda}\right)+\sum_{i=1}^{n} \log \left(1-e^{-\lambda\left(\frac{1-e^{-\alpha x_{i}}}{1+e^{-\alpha x_{i}}}\right)}\right)=0 \\
& \frac{\partial \ell}{\partial \lambda}=\frac{n}{\lambda}-\frac{n \beta e^{-\lambda}}{\left(1-e^{-\lambda}\right)}+(\beta-1) \lambda \sum_{i=1}^{n} \frac{\left(\frac{1-e^{-\alpha x_{i}}}{1+e^{-\alpha x_{i}}}\right) e^{-\lambda\left(\frac{1-e^{-\alpha x_{i}}}{1+e^{-\alpha x_{i}}}\right)}}{\left(1-e^{-\lambda\left(\frac{1-e^{-\alpha x_{i}}}{1+e^{-\alpha x_{i}}}\right)}\right)}-\sum_{i=1}^{n}\left(\frac{1-e^{-\alpha x_{i}}}{1+e^{-\alpha x_{i}}}\right)=0 .
\end{aligned}
$$


For the interval estimate and hypothesis tests of the parameters we required $J(\theta)$ the $3 \times 3$ Fisher information matrix defined by $J(\theta)=-\left\{\partial^{2}(\log \ell(\theta)) / \partial \theta \partial \theta^{T}\right\}$. The approximate of the MLEs of $\theta$, the $\hat{\theta}$, can be approximated as $N_{3}\left(0, J(\hat{\theta})^{-1}\right)$ under usual conditions for parameters in the interior of the parameter space, but not on the boundary. The asymptotic distribution of $\sqrt{n}(\hat{\theta}-\theta)$ is $N_{3}\left(0, J(\hat{\theta})^{-1}\right)$, where $J(\hat{\theta})$ is the unit information matrix evaluated at $\hat{\theta}$, which can be used to construct the approximate confidence interval for each parameter. A $100(1-\epsilon) \%$ asymptotic confidence interval for each parameter $\theta_{r}$ is given by $\mathrm{ACI}_{r}=\left(\hat{\theta}_{r}-Z_{\epsilon / 2} \sqrt{\hat{I}^{r r}}, \hat{\theta}_{r}+Z_{\epsilon / 2} \sqrt{\hat{I}^{r r}}\right)$, where $I^{r r}$ is the $(r, r)$ diagonal element of $I_{n}(\theta)^{-1}$ for $r=1,2,3$ and $Z_{\epsilon / 2}$ is the quantile $(1-\epsilon / 2)$ of the standard normal distribution. The elements of $J(\theta)$ can be obtained from the author under request. The existence and uniqueness of MLEs of a probability models based on some certain sufficient conditions have been considered in various literature by many researchers, the existence and uniqueness of maximum likelihood estimators of the EP was analyzed by Kuş (2007), for the exponential geometric by Adamidis and Loukas (1998), Generalized exponential-power series by Mahmoudi and Jafari (2012), extended exponentialgeometric by Adamidis at el. (2005), recently generalized BurrXII Poisson by Muhammad (2016a) and the complementary exponentiated BurrXII-Poisson by Muhammad (2017) among others. The following theorems provide the existence and uniqueness of the MLEs of the GHLP under some possible conditions with the proofs provided in Appendix B.

Theorem 2. Let $g_{1}\left(\alpha ; \beta, \lambda, x_{i}\right)$ denote the function on the right hand side of the equation (3.2) where $\beta$ and $\lambda$ are the true values of the parameters, then, the equation $g_{1}\left(\alpha ; \beta, \lambda, x_{i}\right)=0$ has at least one root for $\beta \neq 1$ and for $\beta=1$ the root lies in the interval $\left(n /\left\{(2+\lambda / 2) \sum_{i=1}^{n} x_{i}\right\}, n / \sum_{i=1}^{n} x_{i}\right)$.

Theorem 3. Let $g_{2}\left(\beta ; \alpha, \lambda, x_{i}\right)$ denote the function on the right hand side of the equation (3.3) where $\alpha$ and $\lambda$ are the true values of the parameters, then, the equation $g_{2}\left(\beta ; \alpha, \lambda, x_{i}\right)=0$ has at most one root for $\log \left(1-e^{-\lambda}\right)>n^{-1} \sum_{i=1}^{n} \log \left(1-e^{-\lambda\left\{\left(1-e^{-\alpha x_{i}}\right) /\left(1+e^{-\alpha x_{i}}\right)\right)}\right\}$ and is unique.

Theorem 4. Let $g_{3}\left(\lambda ; \alpha, \beta, x_{i}\right)$ denote the function on the right hand side of the equation (3.4) where $\alpha$ and $\beta$ are the true values of the parameters, then, the equation $g_{3}\left(\beta ; \alpha, \lambda, x_{i}\right)=0$ has at least one root for $\beta>1 / 2+(1 / n) \sum_{i=1}^{n}\left\{\left(1-e^{-\alpha x_{i}}\right) /\left(1+e^{-\alpha x_{i}}\right)\right\}$.

\subsection{Simulation study}

Here, we evaluate the performance of the MLEs given by equations (3.2)-(3.4) depending on sample size $n$ for the GHLP distribution. In this process we generated 10,000 samples of size $n=$ $20,30,40,50,100$, and 150 from the GHLP distribution for some various values of $\alpha, \beta$, and $\lambda$. MLEs are obtained by solving the nonlinear equations (3.2)-(3.4) using mlninb in R. The MLEs of $\hat{\alpha}, \hat{\beta}$, and $\hat{\lambda}$ and their standard deviations $\operatorname{sd}(\hat{\alpha}), \operatorname{sd}(\hat{\beta})$, and $\operatorname{sd}(\hat{\lambda})$ of the parameters are given in Table 2 below. The results show that each MLE converges to its true value in all cases when the sample size increases and the standard deviations of the MLEs decrease as the sample size increases.

\section{Real data illustration}

In this section, we provide an application of the GHLP distribution to a real data set. We used the Akaike information criterion (AIC), consistent Akaike information criterion (CAIC), and KolmogorovSmirnov (K-S) test to compare the GHLP and some other existing distributions. The model with the smallest values of these measures fit the data better than the other distributions. The competing distributions are:

- The HLP distribution with cdf given by (1.1). 
Table 2: MLEs and standard deviations for some various values of parameters

\begin{tabular}{|c|c|c|c|c|c|c|c|c|c|}
\hline \multirow{2}{*}{$\begin{array}{c}\text { Sample size } \\
n\end{array}$} & \multicolumn{3}{|c|}{ Actual values } & \multicolumn{3}{|c|}{ Estimated values } & \multicolumn{3}{|c|}{ Standard deviations } \\
\hline & $\alpha$ & $\beta$ & $\lambda$ & $\hat{\alpha}$ & $\hat{\beta}$ & $\hat{\lambda}$ & $\operatorname{sd}(\hat{\alpha})$ & $\operatorname{sd}(\hat{\beta})$ & $\operatorname{sd}(\hat{\lambda})$ \\
\hline \multirow{7}{*}{20} & 0.6 & 1.0 & 0.2 & 0.3484 & 0.6445 & 0.4752 & 0.2779 & 0.7451 & 1.0270 \\
\hline & 1.0 & 1.2 & 1.0 & 1.0751 & 1.4988 & 1.3013 & 0.3698 & 0.8655 & 1.4253 \\
\hline & 0.1 & 0.2 & 0.5 & 0.3826 & 0.1842 & 0.8930 & 0.3908 & 0.0778 & 6.1164 \\
\hline & 0.3 & 0.7 & 0.4 & 0.2615 & 0.6311 & 0.7950 & 0.1282 & 0.4109 & 3.7589 \\
\hline & 2.5 & 1.1 & 2.1 & 3.2405 & 1.2652 & 1.7064 & 1.2870 & 0.6377 & 4.2860 \\
\hline & 1.0 & 1.0 & 1.0 & 1.0914 & 1.2013 & 1.2527 & 0.3901 & 0.5492 & 1.3846 \\
\hline & 0.2 & 0.5 & 1.5 & 0.2606 & 0.5550 & 1.2747 & 0.1173 & 0.1842 & 3.9691 \\
\hline \multirow{7}{*}{30} & 0.6 & 1.0 & 0.2 & 0.7988 & 0.6666 & 0.1397 & 0.2009 & 0.4976 & 0.5541 \\
\hline & 1.0 & 1.2 & 1.0 & 1.0318 & 1.3712 & 1.3004 & 0.3149 & 0.4991 & 1.3962 \\
\hline & 0.1 & 0.2 & 0.5 & 0.3446 & 0.1829 & 0.6371 & 0.3794 & 0.0691 & 2.8381 \\
\hline & 0.3 & 0.7 & 0.4 & 0.2514 & 0.6060 & 0.7650 & 0.1140 & 0.3707 & 3.4513 \\
\hline & 2.5 & 1.1 & 2.1 & 3.0149 & 1.1743 & 1.7622 & 1.0964 & 0.3927 & 2.9208 \\
\hline & 1.0 & 1.0 & 1.0 & 1.0466 & 1.1260 & 1.2523 & 0.3296 & 0.3831 & 1.3701 \\
\hline & 0.2 & 0.5 & 1.5 & 0.2446 & 0.5318 & 1.2913 & 0.0969 & 0.1363 & 1.3302 \\
\hline \multirow{7}{*}{40} & 0.6 & 1.0 & 0.2 & 0.7948 & 0.6463 & 0.1307 & 0.1986 & 0.4601 & 0.5467 \\
\hline & 1.0 & 1.2 & 1.0 & 1.0128 & 1.3138 & 1.2735 & 0.2855 & 0.3892 & 1.3833 \\
\hline & 0.1 & 0.2 & 0.5 & 0.3207 & 0.1835 & 0.5788 & 0.3691 & 0.0661 & 2.0529 \\
\hline & 0.3 & 0.7 & 0.4 & 0.2505 & 0.5911 & 0.7099 & 0.1088 & 0.3443 & 2.5766 \\
\hline & 2.5 & 1.1 & 2.1 & 2.8909 & 1.1424 & 1.8507 & 1.0181 & 0.3141 & 1.5175 \\
\hline & 1.0 & 1.0 & 1.0 & 1.0285 & 1.0942 & 1.2641 & 0.3036 & 0.3070 & 1.3592 \\
\hline & 0.2 & 0.5 & 1.5 & 0.2326 & 0.5216 & 1.3513 & 0.0858 & 0.1104 & 1.3274 \\
\hline \multirow{7}{*}{50} & 0.6 & 1.0 & 0.2 & 0.7946 & 0.6359 & 0.1269 & 0.1964 & 0.4450 & 0.5423 \\
\hline & 1.0 & 1.2 & 1.0 & 1.0037 & 0.6211 & 0.4066 & 0.1727 & 0.5680 & 1.0623 \\
\hline & 0.1 & 0.2 & 0.5 & 0.3088 & 0.1833 & 0.5842 & 0.3647 & 0.0649 & 1.2393 \\
\hline & 0.3 & 0.7 & 0.4 & 0.2477 & 0.5806 & 0.6368 & 0.1044 & 0.3362 & 1.6333 \\
\hline & 2.5 & 1.1 & 2.1 & 2.8522 & 1.1244 & 1.8733 & 0.9546 & 0.2751 & 1.5140 \\
\hline & 1.0 & 1.0 & 1.0 & 1.0144 & 1.0723 & 1.2502 & 0.2831 & 0.2647 & 1.3529 \\
\hline & 0.2 & 0.5 & 1.5 & 0.2270 & 0.5144 & 1.3846 & 0.0798 & 0.0969 & 1.3273 \\
\hline \multirow{7}{*}{100} & 0.6 & 1.0 & 0.2 & 0.7941 & 0.6112 & 0.0926 & 0.1912 & 0.4106 & 0.4077 \\
\hline & 1.0 & 1.2 & 1.0 & 0.9978 & 0.6444 & 0.3096 & 0.1346 & 0.4431 & 0.8330 \\
\hline & 0.1 & 0.2 & 0.5 & 0.3016 & 0.1764 & 0.5559 & 0.3691 & 0.0669 & 0.6401 \\
\hline & 0.3 & 0.7 & 0.4 & 0.2461 & 0.5641 & 0.5677 & 0.0952 & 0.3104 & 1.3488 \\
\hline & 2.5 & 1.1 & 2.1 & 2.6656 & 1.1021 & 2.0777 & 0.8103 & 0.1869 & 1.5002 \\
\hline & 1.0 & 1.0 & 1.0 & 0.9895 & 1.0286 & 1.2264 & 0.2363 & 0.1790 & 1.2841 \\
\hline & 0.2 & 0.5 & 1.5 & 0.1559 & 0.2888 & 0.6507 & 0.0743 & 0.2057 & 1.1702 \\
\hline \multirow{7}{*}{150} & 0.6 & 1.0 & 0.2 & 0.7960 & 0.6006 & 0.0718 & 0.1882 & 0.3997 & 0.3421 \\
\hline & 1.0 & 1.2 & 1.0 & 0.9969 & 0.6429 & 0.3005 & 0.1213 & 0.4317 & 0.8044 \\
\hline & 0.1 & 0.2 & 0.5 & 0.3456 & 0.1667 & 0.4991 & 0.3974 & 0.0720 & 0.5946 \\
\hline & 0.3 & 0.7 & 0.4 & 0.2470 & 0.5562 & 0.4975 & 0.0921 & 0.3005 & 0.8660 \\
\hline & 2.5 & 1.1 & 2.1 & 2.5933 & 1.0939 & 2.1501 & 0.7259 & 0.1521 & 1.4292 \\
\hline & 1.0 & 1.0 & 1.0 & 0.9887 & 1.0200 & 1.1904 & 0.2093 & 0.1483 & 1.1919 \\
\hline & 0.2 & 0.5 & 1.5 & 0.1514 & 0.2810 & 0.6537 & 0.0689 & 0.2018 & 1.1586 \\
\hline
\end{tabular}

- Olapade-generalized half-logistic by Olapade (2014) with $F(x)=1-\left(2^{\beta}\left(1+e^{x / \alpha}\right)^{-\beta}\right)$.

- Power-half-logistic by Krishnarani (2016) with $F(x)=1-2\left(1+e^{\alpha x^{\beta}}\right)^{-1}$.

- The generalized half-logistic with $F(x)=\left(1-e^{-\alpha x}\right)^{\beta}\left(1+e^{-\alpha x}\right)^{-\beta}$. The distribution appears in the study of its estimation procedures by Arora et al. (2010), Kang and Seo (2011), Seo et al. (2012, 2013), and Kantam et al. (2013), where some of its important properties such as the $r^{\text {th }}$-moments, probability weighted moments, and Shannon and Rényi entropies can be obtained from Cordeiro $e t$ al. (2014). 


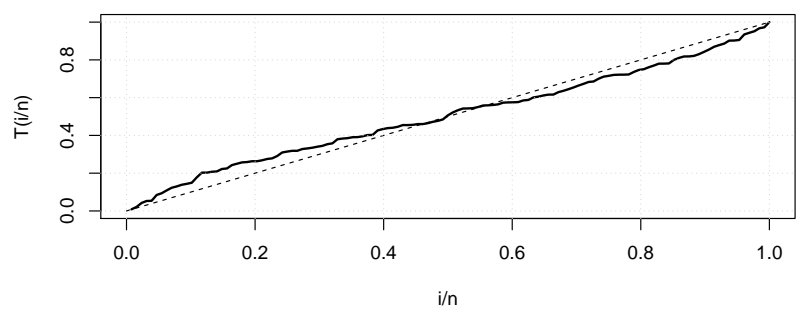

Figure 4: TTT-plot for the remission times (in months) data. TTT = total time on test.

Table 3: MLEs, $\ell(\theta)$, AIC, CAIC, K-S and p-value for the remission times (in months) of bladder cancer patients.

\begin{tabular}{lcccccccc}
\hline \hline Model & $\alpha$ & $\beta$ & $\lambda$ & $\ell(\theta)$ & AIC & CAIC & K-S & $p$-value \\
\hline GHLP & 0.0642 & 1.3909 & 4.4696 & -410.24 & 826.48 & 820.53 & 0.0427 & 0.9737 \\
OGHL & 0.9217 & 0.1250 & - & -412.18 & 828.36 & 824.40 & 0.0661 & 0.6304 \\
PwHL & 0.8880 & 0.2015 & - & -415.10 & 834.19 & 830.22 & 0.0751 & 0.4652 \\
HLP & 0.0574 & - & 3.8836 & -413.17 & 830.34 & 826.37 & 0.0963 & 0.1863 \\
PHL & 0.8880 & - & 0.2015 & -415.10 & 834.19 & 830.22 & 0.0761 & 0.4489 \\
GHL & 0.1440 & 0.9527 & - & -416.64 & 837.27 & 833.30 & 0.0950 & 0.1994 \\
GE & 0.1212 & 1.2180 & - & -413.08 & 830.16 & 826.19 & 0.0725 & 0.5113 \\
EP & 0.3341 & - & 4.3342 & -417.04 & 835.61 & 831.64 & 0.9533 & 0.0000 \\
POEU & 1.5503 & 100 & 6.7528 & -415.15 & 834.30 & 830.33 & 0.0808 & 0.3742 \\
NH & 0.3341 & - & 4.3342 & -417.04 & 838.09 & 820.53 & 0.5709 & 0.0000 \\
HL & 0.1479 & - & - & -416.73 & 835.45 & 833.47 & 0.0989 & 0.1631 \\
\hline \hline
\end{tabular}

- Poisson-half-logistic by Abdel-Hamid (2016) with $F(x)=\left(e^{\lambda\left\{\left(1-e^{-\alpha x}\right) /\left(1+e^{-\alpha x}\right)\right\}}-1\right)\left(e^{\lambda}-1\right)^{-1}$.

- Generalized exponential by Mudholkar and Srivastava (1993) with $F(x)=(1-\exp (-\alpha x))^{\beta}$.

- The EP by Kuş (2007) with $F(x)=\left(e^{\lambda e^{-\alpha x}}-e^{\lambda}\right)\left(1-e^{-\lambda}\right)^{-1}$.

- Poisson-odd exponential uniform by Muhammad (2016b) with $F(x)=\left[1-e^{-\lambda\left(1-e^{-\alpha(x /(\beta-x))}\right.}\right] /\left(1-e^{-\lambda}\right)$.

- Nadarajah and Haghighi (2011) exponential type (NH) with $F(x)=1-e^{1-(1+\lambda x)^{\alpha}}$.

- The half-logistic distribution with cdf as $F(x)=\left(1-e^{-\alpha x}\right)\left(1+e^{-\alpha x}\right)^{-1}$.

The data set is the remission times (in months) of a random sample of 128 bladder cancer patients provided by Lee and Wang (2003).

In Figure 4 the TTT (total time on test)-plot show that the data has upside-down bathtub failure rate function and GHLP distribution has the ability to accommodate upside-down bathtub failure rate curve.

We estimate the unknown parameters of each model by the method of maximum likelihood. The numerical values of the log-likelihood $(\ell(\theta))$, AIC, CAIC, K-S, and its $p$-value obtained are presented in Table 3 below. We also used the muhaz package in $R$ software to obtain the empirical hazard function of the given data set and then fitted with the estimated hazard function of the GHLP obtained using the MLEs in Table 3.

Table 3 indicates that our proposed model the GHLP distribution has the smallest values of the AIC, CAIC, and K-S; thus, GHLP provide better fit than the other competing models. Figure 5 provides the plots of the (i) histogram and estimated density (ii) empirical cdf and estimated cdf of the 


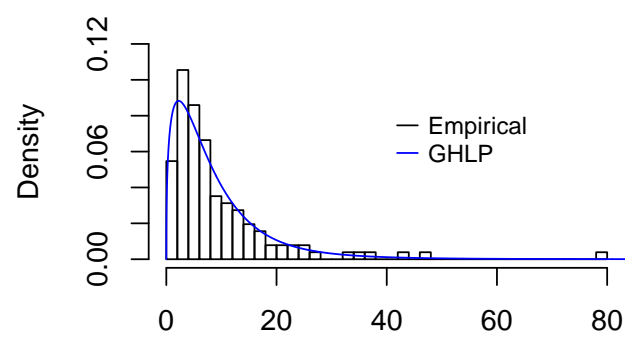

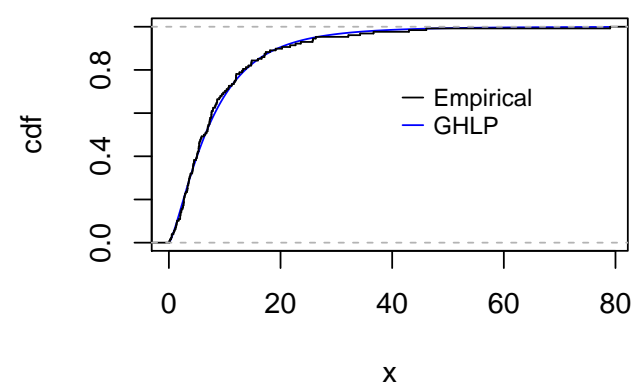

(ii)

Figure 5: Plots of the (i) histogram and estimated density (ii) empirical and estimated cdfs of generalized halflogistic Poisson (GHLP) distribution for the remission times (in months) of bladder cancer patients. cdf $=$ cumulative distribution function.

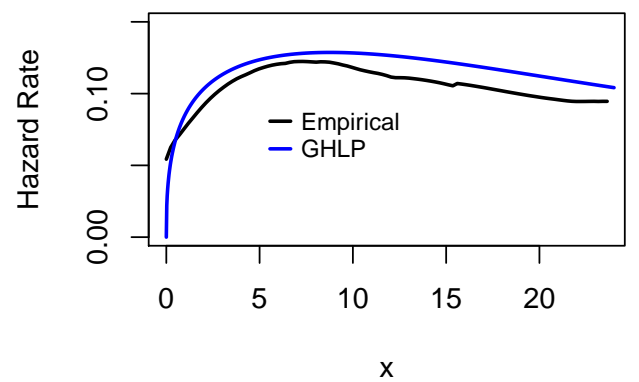

(i)

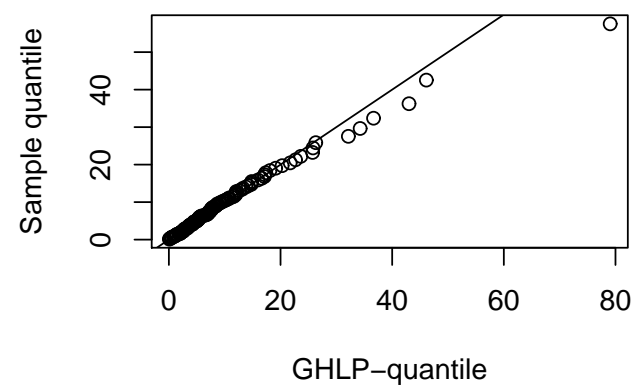

(ii)

Figure 6: Plots of the (i) empirical hazard and estimated hazard (ii) quantile-quantile function of generalized half-logistic Poisson (GHLP) distribution for the remission times (in months) of bladder cancer patients.

GHLP distribution. Figure 6 shows (i) empirical and estimated hazard functions (ii) quantile-quantile of the GHLP distribution for the given data set.

\section{Conclusions}

We introduced a new three-parameter lifetime distribution with increasing, decreasing and upsidedown bathtub-shaped hazard rate functions. We also provide explicit expressions for the $r^{\text {th }}$ ordinary moment, moment generating function, Shannon and Rényi entropies. The estimation of the model parameters was conducted by the maximum likelihood method. The practical significance and applicability of the new distribution are demonstrated in an application to real data, which shows that the GHLP performs better than other existing distributions in terms of fit.

\section{Appendix A:}

Proof of Lemma 1: For (2.6), by applying generalized binomial expanding on

$$
\left(1-e^{-\lambda\left(\frac{1-e^{-\alpha x}}{1+e^{-\alpha x}}\right)}\right)^{J 5}=\sum_{i=0}^{\infty}\left(\begin{array}{c}
J 5 \\
i
\end{array}\right)(-1)^{i} e^{-\lambda i\left(\frac{1-e^{-\alpha x}}{1+e^{-\alpha x}}\right)}
$$


then expanding

$$
e^{-\left(J_{6}+\lambda i\right)\left(\frac{1-e^{-\alpha x}}{1+e^{-\alpha x}}\right)}=\sum_{k=0}^{\infty} \frac{(-1)^{k}\left(J_{6}+\lambda i\right)^{k}}{k !}\left(\frac{1-e^{-\alpha x}}{1+e^{-\alpha x}}\right)^{k}
$$

we get

$$
J\left(J_{1}, J_{2}, J_{3}, J_{4}, J_{5}, J_{6}\right)=\sum_{i=0}^{\infty} \sum_{k=0}^{\infty}\left(\begin{array}{c}
J_{5} \\
i
\end{array}\right) \frac{(-1)^{i+k}\left(\lambda i+J_{6}\right)^{k}}{k !} \int_{0}^{\infty} \frac{x^{J_{1}} e^{-J_{2} x}\left(1-e^{-\alpha x}\right)^{J_{3}+k}}{\left(1+e^{-\alpha x}\right)^{J_{4}+k}} d x .
$$

Let $u=1-e^{-\alpha x}$. By the generalized binomial expansion in the denominator we obtain

$$
\begin{aligned}
J_{\left(J_{1}, J_{2}, J_{3}, J_{4}, J_{5}, J_{6}\right)=} & \sum_{i=0}^{\infty} \sum_{k, l=0}^{\infty}\left(\begin{array}{c}
J_{5} \\
i
\end{array}\right)\left(\begin{array}{c}
-\left(J_{4}+k\right) \\
l
\end{array}\right) \frac{(-1)^{i+k+J_{1}}\left(\lambda i+J_{6}\right)^{k}}{\alpha^{J_{1}+1} k !} \\
& \times \int_{0}^{1} \ln ^{J_{1}}(1-u)(1-u)^{\frac{J_{2}}{\alpha}+l-1} u^{J_{3}+k} d u
\end{aligned}
$$

thus, we have (2.7).

\section{Appendix B:}

Proof of Theorem 2: Let $g_{1}\left(\alpha ; \beta, \lambda, x_{i}\right)$ be the right hand side of (3.2).

For $\beta=1$, let

$$
w_{1}=-2 \sum_{i=1}^{n} \frac{x_{i} e^{-\alpha x_{i}}}{1+e^{-\alpha x_{i}}}-2 \lambda \sum_{i=1}^{n} \frac{x_{i} e^{-\alpha x_{i}}}{\left(1+e^{-\alpha x_{i}}\right)^{2}}
$$

then,

$$
\begin{aligned}
& \lim _{\alpha \rightarrow 0} w_{1}=-\sum_{i=1}^{n} x_{i}-\frac{\lambda}{2} \sum_{i=1}^{n} x_{i} \quad \text { and } \quad \lim _{\alpha \rightarrow \infty} w_{1}=0, \\
& g_{1}\left(\alpha ; \beta, \lambda, x_{i}\right)=\frac{n}{\alpha}-\sum_{i=1}^{n} x_{i}+w_{1}>\frac{n}{\alpha}-\sum_{i=1}^{n} x_{i}+\lim _{\alpha \rightarrow 0} w_{1} \\
& g_{1}\left(\alpha ; \beta, \lambda, x_{i}\right)>0, \quad \text { if } \alpha<\frac{n}{\left(2+\frac{\lambda}{2}\right) \sum_{i=1}^{n} x_{i}} .
\end{aligned}
$$

On the other side

$$
g_{1}\left(\alpha ; \beta, \lambda, x_{i}\right)=\frac{n}{\alpha}-\sum_{i=1}^{n} x_{i}+w_{1}<\frac{n}{\alpha}-\sum_{i=1}^{n} x_{i}+\lim _{\alpha \rightarrow \infty} w_{1}
$$

hence,

$$
g_{1}\left(\alpha ; \beta, \lambda, x_{i}\right)<0, \quad \text { if } \alpha>\frac{n}{\sum_{i=1}^{n} x_{i}}
$$

thus, the root of $g_{1}\left(\alpha ; \beta, \lambda, x_{i}\right)=0$ lies in the interval $\left(n /\left\{(2+\lambda / 2) \sum_{i=1}^{n} x_{i}\right\}, n / \sum_{i=1}^{n} x_{i}\right)$. 
For $\beta \neq 1, \lim _{\alpha \rightarrow 0} g_{1}=\infty$ and $\lim _{\alpha \rightarrow \infty} g_{1}=-\sum_{i=1}^{n} x_{i}<0$, hence, $g_{1}\left(\alpha ; \beta, \lambda, x_{i}\right)$ is a monotone decreasing function from positive to negative; thus, $g_{1}\left(\alpha ; \beta, \lambda, x_{i}\right)=0$ has at least one root.

Proof of Theorem 3: Consider that $\lim _{\beta \rightarrow 0} g_{2}=\infty$, then we show that $\lim _{\beta \rightarrow \infty} g_{2}<0$. But $\lim _{\beta \rightarrow \infty}$ $g_{2}=-n \log \left(1-e^{-\lambda}\right)+\sum_{i=0}^{n} \log \left(1-e^{-\lambda\left(\left(1-e^{-\alpha x_{i}}\right) /\left(1+e^{-\alpha x_{i}}\right)\right)}\right)$; thus, $\lim _{\beta \rightarrow \infty} g_{2}<0$ only if $\log \left(1-e^{-\lambda}\right)>$ $n^{-1} \sum_{i=0}^{n} \log \left(1-e^{-\lambda\left(\left(1-e^{-\alpha x_{i}}\right) /\left(1+e^{-\alpha x_{i}}\right)\right)}\right)$. To prove the uniqueness we show that $g_{2}$ is a decreasing function, that is $g_{2}^{\prime}<0$ and $g_{2}^{\prime}=-n / \beta^{2}<0$.

Proof of Theorem 4: We start with $\lim _{\lambda \rightarrow \infty} g_{3}=-\sum_{i=1}^{n}\left(\left(1-e^{-\alpha x_{i}}\right) /\left(1+e^{-\alpha x_{i}}\right)\right)<0$; therefore, we show that $\lim _{\lambda \rightarrow 0} g_{3}>0$. And $\lim _{\lambda \rightarrow 0} g_{3}=n \beta-n / 2-\sum_{i=1}^{n}\left(\left(1-e^{-\alpha x_{i}}\right) /\left(1+e^{-\alpha x_{i}}\right)\right)$, thus, $\lim _{\lambda \rightarrow 0} g_{3}>$ 0 only if $\beta>1 / 2+(1 / n) \sum_{i=1}^{n}\left(\left(1-e^{-\alpha x_{i}}\right) /\left(1+e^{-\alpha x_{i}}\right)\right)$.

\section{References}

Abdel-Hamid AH (2016). Properties, estimations and predictions for a Poisson-half-logistic distribution based on progressively type-II censored samples, Applied Mathematical Modelling, 40, 7164-7181.

Adamidis K, Dimitrakopoulou T, and Loukas S (2005). On an extension of the exponential-geometric distribution, Statistics \& Probability Letters, 73, 259-269.

Adamidis K and Loukas S (1998). A lifetime distribution with decreasing failure rate, Statistics \& Probability Letters, 39, 35-42.

Ali MM, Pal M, and Woo JS (2007). Some exponentiated distributions, Communications for Statistical Applications and Methods, 14, 93-109.

Arora SH, Bhimani GC, and Patel MN (2010). Some results on maximum likelihood estimators of parameters of generalized half logistic distribution under Type-I progressive censoring with changing failure rate, International Journal of Contemporary Mathematical Sciences, 5, 685698.

Barreto-Souza W and Cribari-Neto F (2009). A generalization of the exponential-Poisson distribution, Statistics \& Probability Letters, 79, 2493-2500.

Chung Y and Kang Y (2014). The exponentiated Weibull-geometric distribution: properties and estimations, Communications for Statistical Applications and Methods, 21, 147-160.

Cordeiro GM, Alizadeh M, and Ortega EM (2014). The exponentiated half-logistic family of distributions: properties and applications, Journal of Probability and Statistics, 2014, 1-21.

Kang SB and Seo JI (2011). Estimation in an exponentiated half logistic distribution under progressively type-II censoring, Communications for Statistical Applications and Methods, 18, 657-666.

Kantam RRL, Ramakrishna V, and Ravikumar MS (2013). Estimation and testing in type I generalized half logistic distribution, Journal of Modern Applied Statistical Methods, 12, 198-206.

Krishnarani SD (2016). On a power transformation of half-logistic distribution, Journal of Probability and Statistics, 2016, 1-10.

Kuş C (2007). A new lifetime distribution, Computational Statistics \& Data Analysis, 51, 4497-4509.

Lee ET and Wang JW (2003). Statistical Methods for Survival Data Analysis (3rd ed), Wiley, New York.

Mahmoudi E and Jafari AA (2012). Generalized exponential-power series distributions, Computational Statistics \& Data Analysis, 56, 4047-4066.

Mudholkar GS and Srivastava DK (1993). Exponentiated Weibull family for analyzing bathtub failure-rate data, IEEE Transactions on Reliability, 42, 299-302.

Muhammad M (2016a). A generalization of the BurrXII-Poisson distribution and its applications, 
Journal of Statistics Applications \& Probability, 5, 29-41.

Muhammad M (2016b). Poisson-odd generalized exponential family of distributions: theory and Applications, Hacettepe University Bulletin of Natural Sciences and Engineering Series B: Mathematics and Statistics, https://doi.org/10.15672/hjms.2016.393.

Muhammad M (2017). The complementary exponentiated BurrXII-Poisson distribution: model, properties and application, Journal of Statistics Applications \& Probability, 6, 33-48.

Muhammad M and Yahaya MA (2017). The half logistic-Poisson distribution, Asian Journal of Mathematics and Applications, 2017, 1-15.

Nadarajah S and Haghighi F (2011). An extension of the exponential distribution, Statistics, 45, 543-558.

Olapade AK (2014). The type I generalized half logistic distribution, Journal of Iranian Statistical Society, 13, 69-82.

Pappas V, Adamidis K, and Loukas S (2015). A generalization of the exponential-logarithmic distribution, Journal of Statistical Theory and Practice, 9, 122-133.

Raja TA and Mir AH (2011). On extension of some exponentiated distributions with application, International Journal of Contemporary Mathematical Sciences, 6, 393-400.

Seo JI, Kim Y, and Kang SB (2013). Estimation on the generalized half logistic distribution under Type-II hybrid censoring, Communications for Statistical Applications and Methods, 20, 63-75.

Seo JI, Lee HJ, and Kan SB (2012). Estimation for generalized half logistic distribution based on records, Journal of the Korean Data and Information Science Society, 23, 1249-1257.

Silva RB, Barreto-Souza W, and Cordeiro GM (2010). A new distribution with decreasing, increasing and upside-down bathtub failure rate, Computational Statistics \& Data Analysis, 54, 935-944.

Silva RB and Cordeiro GM (2015). The Burr XII power series distributions: a new compounding family, Brazilian Journal of Probability and Statistics, 29, 565-589.

Tahmasbi R and Rezaei S (2008). A two-parameter lifetime distribution with decreasing failure rate, Computational Statistics \& Data Analysis, 52, 3889-3901. 